\title{
THERMODYNAMIC PRINCIPLE OF VIRTUAL DISSIPATION AND THE DYNAMICS OF PHYSICAL-CHEMICAL FLUID MIXTURES INCLUDING RADIATION PRESSURE*
}

\author{
BY
}

M. A. BIOT

Royal Academy of Belgium

\begin{abstract}
Equations of motion are obtained for a viscous fluid mixture including thermal and intermolecular diffusion as well as chemical reactions and radiation pressure. They are derived by applying the thermodynamic principle of virtual dissipation. The method also incorporates a new approach to the chemical thermodynamics of open systems which leads to new concepts and formulas for the heat of reaction and the affinity. They are simpler and more general than classical values. Instead of chemical potentials, new "convective potentials" are used which involve physical properties restricted to the system. They do not require extrapolations to absolute zero or the use of undetermined constants. No statistical theory is involved. A noncalorimetric evaluation of the heat of mixing is obtained from the concept of injection pressure of each substance in the mixture. Field equations are derived and a coupling between viscous stress gradients and diffusion is brought out. The convective potentials lead to a new evaluation of the thermodynamic functions of mixtures as well as a new generalized formulation of the Gibbs-Duhem theorem. Translational invariance of the dissipation is discussed and related to total momentum balance. Lagrangian equations of motion with generalized collective coordinates are derived directly from the variational principle and should provide a powerful approach to problems of stellar dynamics with radiation pressure.
\end{abstract}

1. Introduction. Equations of motion and the thermodynamic evolution of a viscous fluid mixture with thermal and intermolecular diffusion have been derived from the principle of virtual dissipation with chemical reactions. Our purpose here is to extend the derivation to a chemically reacting mixture and to include the effect of the radiation pressure at high temperature as well as a number of new and fundamental contributions.

The basic thermodynamics using "convective potentials" instead of chemical potentials which we have introduced for open mixtures is recalled in Sec. 2. Expressions are derived for the heat of mixing in terms of noncalorimetric measurements using the concept of "injection pressure." A new approach to chemical thermodynamics is outlined in Sec. 3 and involves new concepts. In particular, an "intrinsic heat of reaction" is defined which is more representative of the true chemical energy and leads to new evaluations of the heat of reaction, affinities, and chemical potentials, which are both simpler and more general than the classical results.

\footnotetext{
* Received November 24, 1980.
} 
The principle of virtual dissipation is formulated in Sec. 4 for a reacting mixture with inertia forces, viscosity and the thermomolecular diffusion. A simplification is introduced by using a common acceleration for all components of the mixture defined by the barycentric velocity. This also provides further simplication by defining the viscous dissipation in terms of barycentric velocity gradients and viscosity coefficients of the mixture.

With these results, application of the principle of virtual dissipation in Sec. 5 provides the field differential equations of motion and thermodynamic evolution of the mixture. The existence of a novel coupling term between diffusion and the viscous stress gradient is brought out. A simplified expression is obtained for the energy flux, and it is pointed out that in the presence of chemical reactions use of the barycentric velocity is required to obtain physically consistent results. In Sec. 6 the variational procedure leads directly to Lagrangian equations with generalized coordinates.

In Sec. 7 a new and completely general method of evaluation of the thermodynamic functions of the mixture is presented. It is valid for chemically reacting mixtures which are not perfect gases.

A reformulation and generalization of the Gibbs-Duhem theorem is derived in Sec. 8 using the convective potentials instead of the chemical potential. This new result avoids the difficulties due to the presence of undetermined constants in the chemical potentials and the entropy, resulting from the classical formulation.

The translational invariance of the dissipation function for thermomolecular diffusion is discussed in Sec. 9. Along with the new Gibbs-Duhem theorem it shows that the field equations verify the total momentum balance.

The results are directly applicable to gas mixtures at high temperature where the radiation pressure must be taken into account. This is briefly outlined in Sec. 10, and yields a powerful approach to problems of stellar dynamics.

It should be pointed out that the analysis presented here incorporates two distinct features. One is represented by the principle of virtual dissipation which generalizes d'Alembert's principle to non-equilibrium thermodynamics. The other is a restructuring of the thermodynamics of open systems which eliminates fundamental deficiencies of the classical approach of Gibbs. Some of the advantages resulting from these new developments are listed below.

1. By a novel choice of cell and reservoir model including a chemical supply, the entropy and energy for open systems are given new unambiguous definitions as collective concepts based on purely physical operations, without the use of any statistical theory or the introduction of undetermined constants.

2. Gibbs' paradox is avoided within the classical framework.

3. A convective potential is defined which replaces Gibbs' chemical potential. The definition involves only physical properties in the limited range of temperature actually covered by the system. No use is made of physical properties near absolute zero and no undetermined constants have to be considered, in contrast with classical procedures.

4. New concepts in chemical thermodynamics lead to new expressions for the heat of reaction and the affinity which are simpler and more general than classical results.

5. There is no need to distinguish ideal and nonideal systems and results are universally applicable.

6. The new concept of intrinsic heat of reaction is more representative of the true chemical energy than the standard heat of reaction since the latter contains the heat of mixing. 
7. The differential equations of evolution are derived directly from a fundamental physical variational principle expressed by a single scalar relation independent of the coordinate system. This stands in contrast with usual procedures where the variational formulation is obtained from the field differential equations for each particular problem assuming these equations to be known. The method provides a unified approach which brings out the common mathematical structure of a large domain of physical laws and reveals the presence of new terms which had been overlooked.

8. The unified mathematical structure yields an intuitive insight by analogy with simpler familiar phenomena. For example, the mass-spring-dashpot system becomes a universal model for almost all of linear thermodynamics, and includes instabilities by considering negative springs.

9. By using the Lagrangian formulation with generalized coordinates, simplified equations are obtained for the evolution of large complex systems with coupled subsystems of diversified physical nature. These equations are obtained directly from fundamental physical invariants. Finite-element methods are implicit.

10. Considerable conciseness, generality and physical clarity is achieved in this unified thermodynamic approach to mechanics and physical chemistry.

2. Thermodynamics of open mixtures. Before considering the thermochemical system, we shall briefly recall the thermodynamics of open systems presented in an earlier paper [1] for a nonchemical fluid mixture. We shall also add some clarifications regarding some basic physical properties of the new concepts involved.

We consider a "hypersystem" constituted by a primary cell $C_{p}$, supply cells $C_{s k}$, and a thermal well $T W$. The primary cell is characterized by its temperature $T$ and masses $M^{k}$ of pure substances $k$ added to the cell starting from a given initial state. In the present application the primary cell is assumed rigid. The supply cells $C_{s k}$ are large rigid reservoirs each containing a pure substance $k$. They are all at the same pressure and temperature $p_{0}$, $T_{0}$. We have shown that this condition is necessary to avoid Gibbs' paradox. The thermal well is a large rigid isothermal reservoir at the constant temperature $T_{0}$.

Within the hypersystem $C_{p}+\sum_{k} C_{s k}+T W$, matter and heat are transferred to $C_{p}$ from the supply cells and the thermal well by reversible work. Heat is transferred by the use of heat pumps. The reversible process of transferring mass from one cell to the other is called thermobaric transfer. The energy $\mathscr{U}$ and entropy $\mathscr{S}$ of the primary cell are given a new collective definition as the energy and entropy increase of the system $C_{p}+\sum_{k} C_{s k}$.

This definition is justified by the fact that $\mathscr{U}$ and $\mathscr{S}$ are completely determined by the state variables of $C_{p}$, namely its temperature $T$ and the masses $M^{k}$ added to it by thermobaric transfer. Note that $\mathscr{U}$ includes the work of extraction from the supply cells. The work accomplished reversibly on the hypersystem is

$$
\mathscr{V}=\mathscr{U}-T_{0} \mathscr{S}
$$

and is called the primary cell potential. Its differential is

$$
d \mathscr{V}=\sum_{k} \psi_{k} d M^{k}+\theta d s_{T}
$$

where $\theta=T-T_{0}$ is the excess temperature of $C_{p}$ over that of the thermal well. This differential is the work required to inject a mass $d M^{k}$ into $C_{p}$ by thermobaric transfer, and $\theta d s_{T}$ the work required to inject into $C_{p}$ an amount of heat $T d s_{T}$, where $d s_{T}$ is the 
corresponding increase in entropy. The coefficient $\psi_{k}$ is the thermobaric potential. Its value

$$
\psi_{k}=\int_{p_{0} T_{0}}^{p_{k} T}\left(\frac{d p_{k}^{\prime}}{\rho_{k}^{\prime}}+\theta^{\prime} d \bar{s}_{k}\right)
$$

is the work required for the thermobaric transfer of a unit mass of substance $k$. The integral is evaluated along an arbitrary path where the substance injected goes through a variable pressure $p_{k}^{\prime}$, a variable density $\rho_{k}^{\prime}$, a variable temperature $T^{\prime}=\theta^{\prime}+T_{0}$, and increments of specific entropy $d \bar{s}_{k}$. The pressure $p_{k}$ is the pressure at which the substance is injected reversibly into the primary cell. We shall call it the injection pressure. In previous work we have used the term "partial pressure" for $p_{k}$. However, this creates confusion since it is different from the traditional definition which designates as partial pressure the product $p \gamma$ of total pressure $p$ by the molar fraction $\gamma$ of the substance in the mixture. It is only for perfect gases that the two definitions coincide. The traditional definition is physically artificial for mixtures of near-equal properties which cannot be separated by a physical process. In this last case the injection pressures tend to become equal, while the traditional partial pressures do not. This point is closely related to the avoidance of Gibbs' paradox using the physical operational definition of entropy of the present thermodynamic theory.

A relative specific entropy $\bar{s}_{k}$ and a relative specific enthalpy $\bar{\varepsilon}_{k}$ of a substance in the mixture are defined as

$$
\bar{s}_{k}=\int_{p_{0} T_{0}}^{p_{k} T} d \bar{s}_{k}, \quad \bar{\varepsilon}_{k}=\int_{p_{0} T_{0}}^{p_{k} T}\left(\frac{d p_{k}^{\prime}}{\rho_{k}^{\prime}}+T^{\prime} d \bar{s}_{k}\right) .
$$

The characteristic properties of the mixture are embodied in the injection pressure $p_{k}$.

In the process of thermobaric transfer and heat injection the differential of entropy of $C_{p}$ is

$$
d \mathscr{S}=\sum_{k} \bar{s}_{k} d M^{k}+d s_{T} .
$$

Elimination of $d s_{T}$ between Eqs. (2.2) and (2.5) yields

$$
d \mathscr{V}=\sum_{k} \phi_{k} d M^{k}+\theta d \mathscr{S}
$$

where

$$
\phi_{k}=\psi_{k}-\theta \bar{s}_{k}
$$

defines a convective potential. From expressions (2.3) and (2.4) we also obtain

$$
\phi_{k}=\bar{\varepsilon}_{k}-T \bar{s}_{k} .
$$

By contrast with the indeterminacy in traditional procedures, it should be noted that within a given hypersystem the quantities $\phi_{k}, \varepsilon_{k}$ and $\bar{s}_{k}$ are completely defined and do not involve any arbitrary additive constants. This indeterminacy is acknowledged by Gibbs himself [2] and in standard well-known textbooks (Hatsopoulos and Keenan [3], Prigogine and Defay [4]). A further difference from the traditional approach is brought out by noting that the cell energy differential defined as a collective concept is

$$
d \mathscr{U}=\sum_{k} \bar{\varepsilon}_{k} d M^{k}+T d s_{T} .
$$


Elimination of $d s_{T}$ between relations (2.5) and (2.9) using the value (2.8) of $\phi_{k}$ yields

$$
d \mathscr{U}=\sum_{k} \phi_{k} d M^{k}+T d \mathscr{S} .
$$

We see that $\phi_{k}$ is similar to Gibbs' chemical potential $\mu_{k}$. However, (2.10) differs from the classical Gibbs equation by the fact that $\mathscr{U}$ is a collective energy which involves the work of extraction from the supply cells. In addition, $d \mathscr{S}$ is not clearly defined in Gibbs' treatment nor in standard textbooks where it is not explicitly written as (2.5). Finally, Eq. (2.10) constitutes a theorem and is not used to define $\phi_{k}$ as done traditionally when defining the Gibbs chemical potential.

An interesting physical feature of the thermobaric potential $\psi_{k}$ is its definition entirely in terms of reversible work, which does not require the concept of entropy. This stands in contrast with $\phi_{k}$ which involves the entropy. For isothermal transformations $\left(T=T_{0}\right)$, values of $\psi_{k}$ and $\phi_{k}$ are the same. Note that in linear problems for small deviations from equilibrium, relation (2.5) shows that $s_{T}$ becomes a state variable, and in this case the use of $\psi_{k}$ instead of $\phi_{k}$ greatly simplifies the analysis [5].

A continuous mixture may be considered as a collection of open infinitesimal primary cells, called collective primary system. A collective potential for this system is the volume integral.

$$
V=\int_{\Omega}^{\mathscr{V}} \mathscr{V} d \Omega
$$

where $\mathscr{V}$ is the cell potential per unit volume.

For our purpose in the present application we have assumed no volume change of the primary cell. In order to derive some useful relations, a volume change $d v$ of a cell is easily introduced. For example, expression (2.10) becomes

$$
d \mathscr{U}=-p d v+\sum_{k} \phi_{k} d M^{k}+T d \mathscr{S}
$$

by adding the term $-p d v$ where $p$ is the total pressure on the cell. In this case we consider a reversible injection of a mass $d M^{k}$ at constant pressure $p$ and temperature $T$. For such a transformation we write

$$
d s_{T}=\frac{h_{p T}^{k}}{T} d M^{k}
$$

where $h_{p T}^{k} d M^{k}$ is the heat which must be provided to the cell. We have called $h_{p T}^{k}$ the heat of mixing at constant pressure and temperature $[6,7]$. Substitution of the value (2.13) into (2.5) yields

$$
d \mathscr{S}=\sum_{k}\left(\bar{s}_{k}+\frac{h_{p T}^{k}}{T}\right) d M^{k}
$$

This is equivalent to the relation

$$
\left(\frac{\partial \mathscr{S}}{\partial M^{k}}\right)_{p T M}=\bar{s}_{k}+\frac{h_{p T}^{k}}{T}
$$

where the partial derivative is for all variables $p, T$ and masses except $M^{k}$ kept constant. 
It is of interest to show that, using thermodynamic relations, $h_{p T}^{k}$ may be determined without calorimetric measurements. From (2.12) we derive

$$
d(\mathscr{U}+p v-T \mathscr{S})=v d p+\sum_{k} \phi_{k} d M^{k}-\mathscr{S} d T .
$$

This, being an exact differential, implies

$$
\left(\frac{\partial \mathscr{S}}{\partial M^{k}}\right)_{p T M}=-\left(\frac{\partial \phi_{k}}{\partial T}\right)_{p M^{k}} .
$$

The partial derivative on the right side is for constant values of $p$ and all masses $M^{k}$. It may be evaluated by expressing the differential of $\phi_{k}$. From relations (2.4) and (2.8) we derive

$$
d \phi_{k}=\frac{d p_{k}}{\rho_{k}}-\bar{s}_{k} d T \text {. }
$$

When we vary only $T$, maintaining constant $p$ and all values $M^{k}$, we obtain

$$
\left(\frac{\partial \phi_{k}}{\partial T}\right)_{p M_{k}}=\frac{1}{\rho_{k}}\left(\frac{\partial p_{k}}{\partial T}\right)_{p M^{k}}-\bar{s}_{k}
$$

Combining relations (2.15) (2.17) and (2.19) yields

$$
h_{p T}^{k}=-\frac{T}{\rho_{k}}\left(\frac{\partial p_{k}}{\partial T}\right)_{p M^{k}}
$$

Hence the heat of mixing $h_{p T}^{k}$ at constant pressure and temperature is obtained by measuring the injection pressure $p_{k}$ of the substance $k$ as a function of the temperature at constant pressure and constant composition.

For a mixture of perfect gasses the injection pressure is

$$
p_{k}=p \gamma_{k}
$$

where $\gamma_{k}$ is the molar fraction of each component in the mixture. In this case for constant pressure $p$ and constant composition, i.e., constant $\gamma_{k}$, the injection pressure does not vary and therefore

$$
h_{p T}^{k}=0 .
$$

Hence for perfect gas mixtures the heat of mixing at constant pressure and temperature vanishes.

Similarly, we may define a heat of mixing $h_{v T}^{k}$ at constant volume and temperature leading to the value

$$
h_{v T}^{k}=-\frac{T}{\rho_{k}}\left(\frac{\partial p_{k}}{\partial T}\right)_{v M^{k}}
$$

In this case, however, it does not vanish for a mixture of perfect gases.

3. New chemical thermodynamics. When chemical reactions are involved the foregoing procedure of reversible thermobaric and thermal transfer within a hypersystem provides a remarkably simple, rigorous and general treatment of chemical thermodynamics. Since new substances are created by the chemical reaction we must generalize the procedure so that new substances may be produced reversibly within the hypersystem. This is 
accomplished by adjoining to the hypersystem a chemical supply cell $C_{\text {eq }}$ which is a large rigid reservoir where the reaction is in equilibrium at the temperature $T_{\mathrm{eq}}$ and the injection pressures of the pure substances are $p_{\text {keq }}$.

The chemical reaction is measured by a reaction coordinate $\xi$ such that the masses of the various substances produced by the reaction are

$$
d m_{k}=v_{k} d \xi
$$

Since there is no change of the total mass the coefficients $v_{k}$ satisfy the condition

$$
\sum_{k} v_{k}=0
$$

When a reaction $d \xi$ occurs in the rigid primary cell $C_{p}$, without exchange of mass or heat through the boundary, the energy $\mathscr{U}$ of the cell remains constant. We express this by writing

$$
d \mathscr{U}_{\mathrm{ch}}=0
$$

The change of state of the cell due to the reaction is determined by the increase of masses $d m_{k}$ of the reaction and by the condition $d \mathscr{U}_{\mathrm{ch}}=0$ since $d m_{k}$ and $d \mathscr{U}$ are complete state variables of the cell.

While the reaction causes no change of energy of the primary cell, it does produce a change of entropy. We have shown that this entropy may be evaluated by a reversible process which involves only classical concepts by thermobaric and thermal transfers within the hypersystem $[6,7]$. Since new substances are generated, we use the chemical cell $C_{\text {eq }}$ to produce these substances by a reversible process. We consider masses $d m_{k}$ to be produced in $C_{\text {eq }}$ by a reaction $d \xi$. They are referred to as masses "produced" whether positive or negative. They are extracted from $C_{\mathrm{eq}}$ and injected into the primary cell $C_{p}$ by thermobaric transfer. At the same time we inject into $C_{p}$ and $C_{\text {eq }}$ by thermal transfer required amounts of heat so that the energy of these cells does not vary. As a result the state of the cell $C_{\text {eq }}$ remains unchanged. The change of state of $C_{p}$ is determined by the mass increases $d m_{k}$ and the condition of zero increase in energy. Hence the state of the system $C_{p}+C_{\mathrm{eq}}+$ $\sum_{k} C_{s k}$ is now the same as if a reaction $d \xi$ had actually occurred in $C_{p}$ as a closed rigid adiabatic cell, while all other cells remain unchanged. Since the process has occurred reversibly we may evaluate the collective entropy increase $d \mathscr{S}_{\mathrm{ch}}$ by the procedures outlined in the previous section. It is equal and opposite in sign to the entropy increase of the thermal well. Since $d \mathscr{U}_{\mathrm{eq}}=0$, it may also be evaluated from Eq. (2.1), which becomes

$$
d \mathscr{V}_{\mathrm{ch}}=-T_{0} d \mathscr{S}_{\mathrm{ch}}
$$

where $d \mathscr{V}_{\mathrm{ch}}$ is the reversible work performed on the hypersystem.

The quantity $d \mathscr{S}_{\text {ch }}$ is the entropy produced by the reaction as an irreversible process. Hence it is always positive.

It is interesting to note that we have been able to define this entropy in a precise and general way entirely within the framework of the classical thermodynamics of reversible processes.

Following De Donder [8], we put

$$
d \mathscr{S}_{\mathrm{ch}}=\frac{A}{T} d \xi
$$


where $A$ defines the affinity. When a reaction is in equilibrium, $d \mathscr{S}_{\mathrm{ch}}=0$ and $A=0$. If masses $d M^{k}$ and heat $T d s_{T}$ are injected into $C_{p}$ while a reaction $d \xi$ also takes place in it the total increase in entropy $d \mathscr{S}$ of the cell is obtained by adding $d \mathscr{S}_{\text {ch }}$ to the value (2.5). This yields

$$
d \mathscr{S}=\frac{A}{T} d \xi+\sum_{k} \bar{s}_{k} d M^{k}+d s_{T} .
$$

Under the same conditions the increase of energy $d \mathscr{U}$ of the cell is obtained by adding to (2.9) the energy increase $d \mathscr{U}_{\mathrm{ch}}$ due to the chemical reaction. However, according to (3.3), $d \mathscr{U}_{\mathrm{ch}}=0$. Hence

$$
d \mathscr{U}=\sum_{k} \bar{\varepsilon}_{k} d M^{k}+T d s_{T} .
$$

The process of thermobaric transfer provides a very simple method of evaluating the affinity $A$ and leads at the same time to new concepts and results regarding the heat of reaction $[6,7,9,10]$. Consider a reaction $d \xi$ occurring in $C_{p}$ and the opposite reaction $-d \xi$ occurring in $C_{\text {eq }}$. The products $d m_{k}=v_{k} d \xi$ are extracted from $C_{p}$ and injected into $C_{\mathrm{eq}}$ by thermobaric transfer. At the same time an amount of heat $\bar{h}_{p T} d \xi$ is injected into $C_{p}$ so as to maintain constant its temperature $T$. Similarly an amount of heat $-\bar{h}_{p T}^{\text {eq }} d \xi$ is also injected into $C_{\text {eq }}$ so as to maintain constant its temperature $T_{\text {eq }}$. In this process the state of $C_{p}$ and $C_{\text {eq }}$ remains unchanged, hence also their pressures. Applying Eq. (3.6) to $C_{p}$ with $d M^{k}=$ $-v_{k} d \xi$ and $d s_{T}=\left(\bar{h}_{p T} / T\right) d \xi$ we obtain for its entropy increase

$$
d \mathscr{S}=\frac{A}{T} d \xi-\sum_{k} v_{k} \bar{s}_{k} d \xi+\frac{\bar{h}_{p T}}{T} d \xi .
$$

For the chemical supply cell $C_{\text {eq }}$ the reaction is in equilibrium, hence $A=0$. Its entropy increase is therefore

$$
d \mathscr{S}_{\mathrm{eq}}=\sum_{k} v_{k} \bar{s}_{k}^{\mathrm{eq}} d \xi-\frac{\bar{h}_{p T}^{\mathrm{eq}}}{T_{\mathrm{eq}}} d \xi
$$

where $\bar{s}_{k}^{\text {eq }}$ and $\bar{h}_{p T}^{\text {eq }}$ are respectively the values of $\bar{s}_{k}$ and $\bar{h}_{p T}$ in the cell $C_{\text {eq }}$.

In the process no changes occur in the system $C_{p}+C_{\text {eq }}+\sum_{k} C_{s k}$. Hence

$$
d \mathscr{S}+d \mathscr{S}_{\mathrm{eq}}=0 .
$$

We substitute the values (3.8) and (3.9) into Eq. (3.10) and put

$$
\bar{s}_{k}-\bar{S}_{k}^{\mathrm{eq}}=\int_{p_{\mathrm{keq}} T_{\mathrm{eq}}}^{p_{k} T} d \bar{s}_{k} .
$$

Solving the resulting equation for $A / T$ yields

$$
\frac{A}{T}=\sum_{k} v_{k} \int_{p_{k \mathrm{kq}}}^{p_{k} T} d \bar{s}_{k}+\frac{\bar{h}_{p T}^{\mathrm{eq}}}{T_{\mathrm{eq}}}-\frac{\bar{h}_{p T}}{T} .
$$

This is the new expression for the affinity as obtained earlier $[6,7,9,10]$. 
Similar reasoning is applied to the energy increases $d \mathscr{U}$ and $d \mathscr{U}_{\mathrm{eq}}$ of $C_{p}$ and $C_{\mathrm{eq}}$. Applying Eq. (3.7), we write

$$
\begin{aligned}
d \mathscr{U} & =-\sum_{k} v_{k} \bar{\varepsilon}_{k} d \xi+\bar{h}_{p T} d \xi, \\
d \mathscr{U}_{\mathrm{eq}} & =\sum_{k} v_{k} \bar{\varepsilon}_{k}^{\mathrm{eq}} d \xi-\bar{h}_{p T}^{\mathrm{eq}} d \xi,
\end{aligned}
$$

where $\bar{\varepsilon}_{k}^{\text {eq }}$ is the value of $\bar{\varepsilon}_{k}$ in $C_{\text {eq }}$. Again, since no change occurs in the system $C_{p}+C_{\text {eq }}$ $+\sum_{k} C_{s k}$ we put $d \mathscr{U}+d \mathscr{U}_{\text {eq }}=0$. With the values (3.13) and (3.14) this equation yields

$$
\bar{h}_{p T}-\bar{h}_{p}^{\mathrm{eq}}=\sum_{k} v_{k} \int_{p_{\mathrm{keq}} T_{\mathrm{eq}}}^{p_{k} T} d \bar{\varepsilon}_{k}
$$

We may express the affinity by eliminating $\bar{h}_{p T}$ between equations (3.12) and (3.15). Hence

$$
A=\sum_{k} v_{k} \int_{p_{k \mathrm{keq}} T_{\mathrm{eq}}}^{p_{k} T}\left(T d \bar{s}_{k}-d \bar{\varepsilon}_{k}\right)+\bar{h}_{p}^{\mathrm{eq}} T\left(\frac{T}{T_{\mathrm{eq}}}-1\right) .
$$

Relations (3.15) and (3.16) also coincide with the results derived earlier $[6,7,9,10]$.

The quantity $\bar{h}_{p}$ is a new concept which we have called the intrinsic heat of reaction. It is the heat of reaction when it takes place at constant pressure temperature and composition, the masses produced (positive or negative) being extracted as the reaction proceeds. It is more representative of the true chemical energy than the standard heat of reaction at constant pressure and temperature since the latter includes the heat of mixing.

Note that the intrinsic heat of reaction $\bar{h}_{p T}$ by its very definition is the heat of reaction, not only at constant composition pressure and temperature but also at constant volume. Hence if we denote by $\bar{h}_{v T}$ the intrinsic heat of reaction at constant volume and temperature we may write

$$
\bar{h}_{v T}=\bar{h}_{p T} .
$$

It is easily seen that the standard heat of reaction $h_{p T}$ at constant pressure and temperature is given by

$$
h_{p T}=\bar{h}_{p T}+\sum_{k} v_{k} h_{p T}^{k}
$$

where $h_{p T}^{k}$ is the heat of mixing. Similarly, the standard heat of reaction at constant volume and temperature is

$$
h_{v T}=\bar{h}_{v T}+\sum_{k} v_{k} h_{v T}^{k} .
$$

Both $h_{p T}^{k}$ and $h_{v T}^{k}$ are given by expressions (2.20) and (2.23) which do not involve calorimetric measurements.

In differential form we may write (3.15) as

$$
d \bar{h}_{p T}=\sum_{k} v_{k} d \bar{\varepsilon}_{k} .
$$

This new result $[6,7]$ yields the intrinsic heat of reaction when both temperature and composition are varied. It is more general than Kirchhoff's classical expression of the standard heat of reaction which considers only temperature variations. It differs also fundamentally from the classical result by the fact that $\bar{\varepsilon}_{k}$ is defined by the properties of the pure reactants and the injection pressures. 
An expression for the affinity formally similar to the classical result may be obtained by introducing the concept of chemical potential in a novel way. We assume that we may write

$$
\begin{aligned}
& \bar{h}_{p T}=\sum_{k} v_{k}\left[\int_{0}^{p_{k} T} d \bar{\varepsilon}_{k}+\bar{\varepsilon}_{k}(0)\right], \\
& \frac{\bar{h}_{p T}^{\text {eq }}}{T_{\text {eq }}}=\sum_{k} v_{k}\left[\int_{0}^{p_{k e q} T_{\text {eq }}} d \bar{s}_{k}+\bar{s}_{k}(0)\right],
\end{aligned}
$$

where the lower limit of integration implies an extrapolation to the solid state at absolute zero, while $\bar{\varepsilon}_{k}(0)$ and $\bar{s}_{k}(0)$ are constants characteristic of the pure substances and independent of the particular reaction involved. With the values (3.21) and (3.22) Eq. (3.12) yields the affinity in the form

$$
A=-\sum v_{k} \mu_{k}
$$

where we have put

$$
\mu_{k}=\int_{0}^{p_{k} T}\left(d \bar{\varepsilon}_{k}-T d \bar{s}_{k}\right)+\bar{\varepsilon}_{k}(0)-T \bar{s}_{k}(0)
$$

as a new definition of the chemical potential $[9,10]$. ( $T$ is constant in the integration.)

Eqs. (3.21) and (3.22) may be considered as additional axioms while the constants $\bar{\varepsilon}_{k}(0)$ and $\bar{s}_{k}(0)$ are determined experimentally from chemical reactions. These values may also be derived from quantum statistical mechanics (Fowler and Guggenheim [11]) but this is rarely practical and involves a large and ponderous additional branch of science. In the case of validity of Nernst's principle we may put $\bar{s}_{k}(0)=0$.

Using the affinity we may write Eq. (3.4) in the form

$$
d \mathscr{V}_{\mathrm{ch}}=-A d \xi+\theta d \mathscr{S}_{\mathrm{ch}} .
$$

By adding this expression to Eq. (2.6) we obtain

$$
d \mathscr{V}=-A d \xi+\cdot \sum_{k} \phi_{k} d M^{k}+\theta d \mathscr{S}
$$

where $d \mathscr{S}$ is now given by (3.6). This value of $d \mathscr{V}$ is the differential of the cell potential when masses $d M^{k}$ and heat are injected into the cell while a chemical reaction occurs at the same time.

The state variables of the primary cell are now $\xi, M^{k}, \mathscr{S}$ where $M^{k}$ are the masses added to the cell by convection as distinct from those added by the chemical reaction. The cell potential is a function of these variables

$$
\mathscr{V}=\mathscr{V}\left(\xi, M^{k}, \mathscr{S}\right)
$$

and leads to the fundamental relations

$$
\partial \mathscr{V} / \partial \xi=-A, \quad \partial \mathscr{V} / \partial M^{k}=\phi_{k}, \quad \partial \mathscr{V} / \partial \mathscr{S}=\theta .
$$

This may be generalized to the case where several reactions take place. For each reaction we write

$$
d m_{k}=v_{k \rho} d \xi_{\rho}
$$


The values (3.6) and (3.26) become

$$
\begin{aligned}
d \mathscr{S} & =\sum_{\rho} \frac{A_{\rho}}{T} d \xi_{\rho}+\sum_{k} \bar{s}_{k} d M^{k}+d s_{T}, \\
d \mathscr{V} & =-\sum_{\rho} A_{\rho} d \xi_{\rho}+\sum_{k} \phi_{k} d M^{k}+\theta d \mathscr{S} .
\end{aligned}
$$

Elimination of $d s_{T}$ between (3.7) and (3.30) also yields

$$
d \mathscr{U}=-\sum_{\rho} A_{\rho} d \xi_{\rho}+\sum_{k} \phi_{k} d M^{k}+T d \mathscr{S}
$$

4. Principle of virtual dissipation for a chemically reacting system. The principle of virtual dissipation was formulated in the context of a fluid mixture occupying a domain $\Omega$ and expressed as [1]

$$
\sum_{i} I_{i} \delta q_{i}+\int_{\Omega}\left(\delta_{r} \mathscr{V}+\mathscr{G} \delta \rho+T \delta s^{*}\right) d \Omega=0
$$

It remains formally the same when chemical reactions are involved. In this equation $\mathscr{V}$ is the cell potential of the mixture per unit volume. It is a function of $\xi_{\rho}, M^{k}$ and $\mathscr{S}$, where $\xi_{\rho}$ is the reaction coordinate of each reaction for masses produced per unit volume, while $M^{k}$ and $\mathscr{S}$ are masses added and entropy, also per unit volume. The system is in a gravity field of potential $\mathscr{G}$ and $\rho$ is the density of the mixture. The terms $\sum_{i} I_{i} \delta q_{i}$ represent the virtual work of the inertia forces.

As before, we write

$$
\mathscr{S}=s^{*}+s, \quad s=-\partial S_{i} / \partial x_{i}
$$

where $S_{i}$ is the entropy transferred per unit area and $s^{*}$ is the entropy produced per unit volume with initial conditions $S_{i}=s=s^{*}=0$. The summation convention is used for vectors and tensors. The entropy flux is

$$
\dot{S}_{i}=\sum_{k} \bar{S}_{k} \dot{M}_{i}^{k}+\dot{S}_{i}^{t}
$$

The dot indicates a time derivative. The rate of mass flow of each substance is $\dot{M}_{k}$ while $\dot{S}_{i}^{t}$ is the entropy flux due to the heat flux. Hence $s$ is the entropy supplied by convection and conduction (see below).

Mass conservation is expressed by

$$
M^{k}=-\partial M_{i}^{k} / \partial x_{i}
$$

where $M^{k}$ is the mass of each substance supplied by convection to the unit volume and $M_{i}^{k}$ is the total mass which has flowed through a unit area, with initial conditions $M^{k}=M_{i}^{k}=0$. The density of the mixture may be written as

$$
\rho=m_{0}-\sum_{k} \frac{\partial M_{i}^{k}}{\partial x_{i}}
$$

where $m_{0}$ is the initial density.

In (4.1) we vary $\delta \xi_{\rho}, \delta M_{i}^{k}$ and $\delta S_{i}$. The variation $\delta s^{*}$ is also expressed linearly in terms of these variations. However, the variations are subject to two restrictions. Although the 
actual system may exchange heat and mass through the boundary we assume that for the variations there is no such exchange. In addition, when varying $\delta \mathscr{S}$ in $\delta \mathscr{V}$ we put $\delta s^{*}=0$. This is indicated by the symbol $\delta_{r}$.

Consider first the chemical reactions. The local reaction coordinates $\xi_{\rho}$ define the mass $d m_{k}$ of substance $k$ produced per unit volume through the relation

$$
d m_{k}=\sum_{\rho} v_{k \rho} d \xi_{\rho}
$$

The rate of dissipation due to the chemical reactions is

$$
T s^{*}=\sum_{\rho} A_{\rho} \dot{\xi}_{\rho}
$$

since $\left(A_{\rho} / T\right) \dot{\xi}_{\rho}$ is the rate of entropy produced by each reaction. In analogy with mechanical problems where dissipative stresses are expressed in terms of rates and velocities by taking into account kinetic properties, we shall express $A_{\rho}$ in terms of reaction rates thus introducing the chemical kinetics. We may write

$$
\partial \mathscr{V} / \partial \xi_{\rho}=-A_{\rho}\left(\xi_{\sigma}, M^{k}, \mathscr{S}\right)
$$

as a consequence of Eq. (3.31). Hence $A_{\rho}$ is a known function of the state variables $\xi_{\sigma}, M^{k}$, and $\mathscr{S}$. We shall assume that the rates of various chemical reactions may be derived from chemical kinetics and are completely determined by the local state variablis $\xi_{\sigma}, M^{k}, \mathscr{S}$ of the mixture. Hence we write

$$
\dot{\xi}_{\rho}=f_{\rho}\left(\xi_{\sigma}, M^{k}, \mathscr{S}\right)
$$

The possible influence of other rate variables on the reaction rates is therefore assumed to be negligible. We solve Eqs. (4.9) for $\xi_{\sigma}$ and substitute these values in $A_{\rho}$. We find

$$
A_{\rho}=\mathscr{R}_{\rho}\left(\dot{\xi}_{\sigma}, M^{k}, \mathscr{S}\right) \text {. }
$$

The affinity has thus become a rate function $\mathscr{R}_{\rho}$ of $\dot{\xi}_{\sigma}$. The rate of dissipation due to chemical reactions may now be written

$$
T s^{*}=\sum_{\rho} \mathscr{R}_{\rho} \dot{\xi}_{\rho} .
$$

In this form it embodies the chemical kinetics and is analogous to familiar expressions in mechanics when the rate of dissipation is evaluated in terms of velocities and viscosity coefficients. The corresponding virtual dissipation due to the chemical reactions is

$$
T \delta s^{*}=\sum_{\rho} \mathscr{R}_{\rho} \delta \xi_{\sigma} .
$$

This virtual dissipation must be completed by additional terms due to thermomolecular diffusion and viscosity. They have been derived previously [1].

Per unit volume, the dissipation function $\mathscr{D}_{\mathrm{tm}}$ due to thermomolecular diffusion was found to be

$$
\mathscr{D}_{\mathrm{tm}}=\frac{1}{2} \sum_{l k i} \mathscr{C}^{l k} \dot{M}_{i}^{l} \dot{M}_{i}^{k}+\sum_{k i} \mathscr{C}^{k} \dot{M}_{i}^{k} \dot{S}_{i}+\frac{T}{2 k} \sum_{i}\left(\dot{S}_{i}\right)^{2}
$$

The coefficients are functions of the local state variables. It is shown in Sec. 7 how they may be derived to obey the condition of invariance under a translation. As pointed out, the 
quadratic form (4.13) implies the local validity of Onsager's principle for thermomolecular diffusion $[12,13]$. The virtual dissipation due to thermomolecular diffusion is (with summation convention)

$$
T d s^{*}=\sum_{k} \frac{\partial \mathscr{D}_{\mathrm{tm}}}{\partial \dot{M}_{i}^{k}} \delta M_{i}^{k}+\frac{\partial \mathscr{D}_{\mathrm{tm}}}{\partial \dot{S}_{i}} \delta S_{i} .
$$

The dissipation function due to the viscosity was also evaluated taking into account intermolecular viscosity effects [1]. However, in practice is seems useful to introduce a simplification which requires only a knowledge of two overall viscosity coefficients of the mixture. This is obtained by considering the barycentric velocity $v_{i}$ defined by the equations

$$
v_{i}=\dot{M}_{i} / \rho, \quad \dot{M}_{i}=\sum_{k} \dot{M}_{i}^{k}
$$

We put

$$
v_{i j}=\partial v_{i} / \partial x_{j} .
$$

The dissipative stress in the mixture due to viscosity is then expressed as

$$
\sigma_{i j}=\sigma_{j i}=\eta\left(v_{i j}+v_{j i}\right)+\lambda \delta_{i j} v_{i i}
$$

where $\eta$ and $\lambda$ are viscosity coefficients of the mixture, functions of the local state variables, and $\delta_{i j}$ is the unit tensor. It may be written

$$
\sigma_{i j}=\partial \mathscr{D}_{\mathrm{v}} / \partial v_{i j}
$$

where the quadratic form in $v_{i j}$

$$
\mathscr{D}_{\mathrm{v}}=\frac{1}{2} \sigma_{i j} v_{i j}
$$

is the dissipation function due to viscosity. The corresponding virtual dissipation is

$$
T \delta s^{*}=\sigma_{i j} \delta a_{i j}
$$

where

$$
\delta a_{i j}=\frac{\partial}{\partial x_{j}}\left(\frac{1}{\rho} \sum_{k} \delta M_{i}^{k}\right)
$$

The total virtual dissipation due to the variations $\delta M_{i}^{k}, \delta S_{i}$ and $\delta \xi_{\rho}$ is the sum of the values (4.12) (4.14) and (4.20). Its value is

$$
T \delta s^{*}=\sigma_{i j} \delta a_{i j}+\sum_{k} \frac{\partial \mathscr{D}_{\mathrm{tm}}}{\partial \dot{M}_{i}^{k}} \delta M_{i}^{k}+\frac{\partial \mathscr{D}_{\mathrm{tm}}}{\partial \dot{S}_{i}} \delta S_{i}+\sum_{\rho} \mathscr{R}_{\rho} d \xi_{\rho}
$$

Comparing the terms $\sigma_{i j} \delta a_{i j}$ and $\mathscr{R}_{\rho} \delta \xi_{\rho}$, it is interesting to note that the dissipative stress $\sigma_{i j}$ is the tensor equivalent of the affinity $\mathscr{R}_{\boldsymbol{\rho}}$.

Finally we must also evaluate the virtual work of the inertia forces. We introduce the simplifying assumption that the acceleration of each component in the mixture is equal to its barycentric acceleration

$$
a_{i}=\frac{\partial v_{i}}{\partial t}+v_{j} \frac{\partial v_{i}}{\partial x_{j}}
$$


The virtual work of the inertia forces is then

$$
\sum_{j} I_{j} \delta q_{j}=\int_{\Omega} \sum_{k} m_{k} a_{i} \frac{\delta M_{i}^{k}}{m_{k}} d \Omega=\int_{\Omega} a_{i} \sum_{k} \delta M_{i}^{k} d \Omega
$$

where $m_{k}$ is the mass of the component $k$ per unit volume. Hence we are neglecting all local accelerations due to the relative motion in a system in translation with the local barycentric velocity. This is consistent with the assumption of local validity of Onsager's principle for viscous and diffusive forces, since this requires the Coriolis forces due to the relative motion to be negligible [14].

Physical significance of the heat flux. In expression (4.3) for the entropy flux we may put $\dot{S}_{i}^{t}=\dot{H}_{i} / T$ where $\dot{H}_{i}$ is the heat flux. It represents the heat which must be injected across a surface, in addition to the mass flux $\dot{M}_{i}^{k}$, in order to achieve the same change of state as due to the actual flow of the mixture. It includes what is commonly called the heat of transport.

Alternative formulations. In the principle of virtual dissipation (4.1), $\delta_{r} \mathscr{V}$ is a restricted variation such that $\delta \mathscr{S}=-\partial \delta S_{i} / \partial x_{i}$. Since $\delta S_{i}=0$ at the boundary we have

$$
\begin{aligned}
\int_{\Omega} \delta_{r} \mathscr{V} d \Omega & =\int_{\Omega}\left(\sum_{k} \phi_{k} \delta M^{k}-\theta \frac{\partial}{\partial x_{i}} \delta S_{i}\right) d \Omega \\
& =\int_{\Omega}\left(\sum_{k} \phi_{k} \delta M^{k}-T \frac{\partial}{\partial x_{i}} \delta S_{i}\right) d \Omega=\int_{\Omega} \delta_{r} \mathscr{U} d \Omega
\end{aligned}
$$

and (4.1) takes the form

$$
\sum_{i} I_{i} \delta q_{i}+\int_{\Omega}\left(\delta_{r} \mathscr{U}+\mathscr{G} \delta \rho+T \delta s^{*}\right) d \Omega=0
$$

On the other hand, for a system at constant temperature $T=T_{0}$ or near equilibrium the principle (4.1) becomes

$$
\sum_{i} I_{i} \delta q_{i}+\int_{\Omega}\left(\delta \mathscr{V}+\mathscr{G} \delta \rho+T_{0}\right) d \Omega=0
$$

where the variation is unrestricted and due only to $\delta M^{k}$.

5. Variational derivation of the dynamical field equations for a physical-chemical fluid mixture. The principle of virtual dissipation (4.1) has been used to derive the equations of motion of a viscous fluid mixture with thermomolecular diffusion [1]. This derivation will be extended to a chemically reacting mixture.

The components of the mixture undergo multiple chemical reactions measured by the reaction coordinates $\xi_{\rho}$. The state of the mixture is determined by the scalar fields $\mathscr{S}, M^{k}, \xi_{\rho}$ or equivalently by the vector fields $S_{i}, M_{i}^{k}$ and the scalar fields $\xi_{\rho}$ and $s^{*}$. We substitute in (4.1) the value (4.24) for the virtual work of the inertia forces, and put $T \delta s^{*}$ equal to (4.22). We vary $\delta M_{i}^{k}, \delta S_{i}$ and $\delta \xi_{\rho}$ arbitrarily inside the domain $\Omega$. We proceed as earlier [1] taking into account the holonomic constraints (4.2) (4.4) and (4.5), namely

$$
\delta \rho=-\sum_{k} \frac{\partial}{\partial x_{i}}\left(\delta M_{i}^{k}\right), \quad \delta_{r} \mathscr{S}=\delta s=-\frac{\partial}{\partial x_{i}}\left(\delta S_{i}\right), \quad \delta M^{k}=-\frac{\partial}{\partial x_{i}}\left(\delta M_{i}^{k}\right),
$$


and integrating by parts. This yields

$$
\begin{gathered}
a_{i}+\frac{\partial}{\partial x_{i}}\left(\frac{\partial \mathscr{V}}{\partial M^{k}}+\mathscr{G}\right)-\frac{1}{\rho} \frac{\partial}{\partial x_{j}}\left(\frac{\partial \mathscr{D}_{\mathrm{v}}}{\partial v_{i j}}\right)+\frac{\partial \mathscr{D}_{\mathrm{tm}}}{\partial \dot{M}_{i}^{k}}=0, \\
\frac{\partial}{\partial x_{i}}\left(\frac{\partial \mathscr{V}}{\partial \mathscr{S}}\right)+\frac{\partial \mathscr{D}_{\mathrm{tm}}}{\partial \dot{S}_{i}}=0, \quad \frac{\partial \mathscr{V}}{\partial \xi_{\rho}}+\mathscr{R}_{\rho}=0 .
\end{gathered}
$$

To complete these field equations we must introduce an additional equation for the entropy produced $s^{*}$ by expressing the rate of dissipation as

$$
T s^{*}=2 \mathscr{D}_{\mathrm{tm}}+2 \mathscr{D}_{\mathrm{v}}+\sum_{\rho} A_{\rho} \dot{\xi}_{\rho}
$$

Eqs. (5.2) bring out a fundamental symmetry in the mathematical structure of the field equations. This is further illustrated by assuming the chemical reactions to be sufficiently close to local equilibrium, so that we may apply Onsager's principle, by writing

$$
\mathscr{R}_{\rho}=\frac{\partial \mathscr{D}_{\mathrm{ch}}}{\partial \dot{\xi}_{\rho}}
$$

where

$$
\mathscr{D}_{\mathrm{ch}}=\frac{1}{2} \sum_{\sigma \rho} B_{\sigma \rho} \dot{\xi}_{\sigma} \dot{\xi}_{\rho}
$$

is a dissipation function for the chemical reactions and $B_{\sigma \rho}$ are functions of the local state. We may now write the field equations as

$$
\begin{gathered}
a_{i}+\frac{\partial}{\partial x_{i}}\left(\frac{\partial \mathscr{V}}{\partial M^{k}}+\mathscr{G}\right)-\frac{1}{\rho} \frac{\partial}{\partial x_{j}}\left(\frac{\partial \mathscr{D}}{\partial v_{i j}}\right)+\frac{\partial \mathscr{D}}{\partial \dot{M}_{i}^{k}}=0, \\
\frac{\partial}{\partial x_{i}}\left(\frac{\partial \mathscr{V}}{\partial \mathscr{S}}\right)+\frac{\partial \mathscr{D}}{\partial \dot{S}_{i}}=0, \quad \frac{\partial \mathscr{V}}{\partial \xi_{\rho}}+\frac{\partial \mathscr{D}}{\partial \dot{\xi}_{\rho}}=0
\end{gathered}
$$

with a single dissipation function

$$
\mathscr{D}=\mathscr{D}_{\mathrm{v}}+\mathscr{D}_{\mathrm{tm}}+\mathscr{D}_{\mathrm{ch}} .
$$

This dissipation function may even be generalized by including cross-products of the type $\left(\partial v_{i} / \partial x_{i}\right) \xi_{\rho}$ which imply that an additional entropy is produced by the simultaneous action of a bulk rate and a reaction rate. It should be noted that this effect is different from the chemical relaxation associated with internal coordinates.

Note that Eqs. (5.6) are directly applicable to linearized perturbations propagating as acoustic waves in a fluid reacting mixture in initial equilibrium. The treatment is entirely similar to the linear thermodynamic analysis presented elsewhere for the more general case of the initially stressed solid [5]. It was shown how the treatment may be simplified by using the variables $\psi_{k}$ and $s_{T}$ instead of $\phi_{k}$ and $\mathscr{S}$.

The field equations (5.2) may be written in more explicit form by substituting the values

$$
\frac{\partial \mathscr{V}}{\partial \xi_{\rho}}=-A_{\rho}, \quad \frac{\partial \mathscr{V}}{\partial M^{k}}=\phi_{k}, \quad \frac{\partial \mathscr{V}}{\partial \mathscr{S}}=\theta
$$


derived from the differential (3.31). Also by definition of $\mathscr{R}_{\rho}$, the following equations

$$
\frac{\partial \mathscr{V}}{\partial \xi_{\rho}}+\mathscr{R}_{\rho}=0
$$

are equivalent to Eqs. (4.9) for the rate of reaction. Hence the field equations (5.2) may be written as

$$
a_{i}+\frac{\partial \varphi_{k}}{\partial x_{i}}-\frac{1}{\rho} \frac{\partial \sigma_{i j}}{\partial x_{j}}+\frac{\partial \mathscr{D}_{\mathrm{tm}}}{\partial \dot{M}_{i}^{k}}=0, \quad \frac{\partial \theta}{\partial x_{i}}+\frac{\partial \mathscr{D}_{\mathrm{tm}}}{\partial \dot{S}_{i}}=0, \quad \dot{\xi}_{\rho}=f_{\rho},
$$

where $\varphi_{k}=\phi_{k}+\mathscr{G}$.

By proceeding as earlier [1] we derive an energy flux theorem. We add Eqs. (5.2) after multiplying the first set by $\dot{M}_{i}^{k}$, the second set by $\dot{S}_{i}$, and the third by $\dot{\xi}_{\rho}$. After some algebraic manipulation this yields

$$
\dot{M}_{i} a_{i}+\frac{\partial}{\partial x_{i}}\left(-v_{j} \sigma_{j i}+\sum_{k} \dot{M}_{i}^{k} \varphi_{k}+T \dot{S}_{i}\right)+\dot{\mathscr{U}}+\mathscr{G} \dot{\rho}=0 .
$$

In addition we may write

$$
\dot{M}_{i} a_{i}=\dot{M}_{i} \frac{\partial v_{i}}{\partial t}+\frac{1}{2} \dot{M}_{i} \frac{\partial v^{2}}{\partial x_{i}}
$$

where $v^{2}=v_{i} v_{i}$. Since $\dot{M}_{i}=\rho v_{i}$, taking into account conservation of mass, we obtain

$$
\dot{M}_{i} a_{i}=\frac{1}{2} \frac{\partial}{\partial t}\left(\rho v^{2}\right)+\frac{1}{2} \frac{\partial}{\partial x_{i}}\left(\dot{M}_{i} v^{2}\right)
$$

With this value Eq. (5.11) becomes

$$
\frac{\partial F_{i}}{\partial x_{i}}+\frac{1}{2} \frac{\partial}{\partial t}\left(\rho v^{2}\right)+\dot{\mathscr{U}}+\dot{\rho} \mathscr{G}=0
$$

where

$$
F_{i}=\frac{1}{2} \dot{M}_{i} v^{2}-v_{j} \sigma_{i j}+\sum_{i} \dot{M}_{i}^{k} \varphi_{k}+T \dot{S}_{i}
$$

represents the energy flux. Notice that this implies that the kinetic energy per unit volume is $\frac{1}{2} \rho v^{2}$. Hence it is assumed that the kinetic energy of the components relative to barycentric motion is negligible. This approximation is a consequence of the use of the barycentric acceleration $a_{i}$ in Eqs. (5.2) instead of the actual acceleration of each component as done in the earlier paper [1] for the case of a non-reacting mixture. It can be verified that in the presence of chemical reactions it is essential to use the barycentric acceleration in order to avoid spurious but negligible terms in the energy balance equation (5.14). This is physically consistent with the fact that the chemical kinetics we have adopted is for reactants without relative average transport velocities.

6. Lagrangian equations. The principle of virtual dissipation (4.1) leads directly to Lagrangian equations for a chemically reacting mixture. We put

$$
M_{j}^{k}=M_{j}^{k}\left(q_{i}, x_{l}, t\right), \quad S_{j}=S_{j}\left(q_{i}, x_{l}, t\right), \quad \xi_{\rho}=\xi_{\rho}\left(q_{i}, x_{l}, t\right),
$$


where $q_{i}$ are unknown generalized coordinates to be determined as functions of time. We also write the entropy produced as

$$
s^{*}=s^{*}\left(q_{i}^{\prime}, x_{l}, t\right)
$$

where $q_{i}^{\prime}$ are auxiliary generalized coordinates. When substituting these values into the variational equations (4.1) we vary only $q_{i}$ and evaluate the corresponding variations $\delta M_{j}^{k}$, $\delta S_{j}, \delta \xi_{\rho}$. We proceed as earlier [1]. In the presence of reactions the virtual dissipation contains an additional term

$$
T \delta s^{*}=\int_{\Omega} \sum_{\rho} \mathscr{R}_{\rho} \delta \xi_{\rho} d \Omega=R_{i} \delta q_{i}
$$

where

$$
R_{i}=\int_{\Omega} \sum_{\rho} \mathscr{R}_{\rho} \frac{\partial \xi_{\rho}}{\partial q_{i}} d \Omega
$$

represents a generalized affinity. With the barycentric acceleration the generalized inertia forces are

$$
I_{i}=\int_{\Omega} a_{j} \frac{\partial M_{j}}{\partial q_{i}} d \Omega
$$

where $M_{i}=\sum_{k} M_{i}^{k}$. From these values we derive the Lagrangian equations

$$
I_{i}+R_{i}+\frac{\partial D}{\partial \dot{q}_{i}}+\frac{\partial \mathscr{P}}{\partial q_{i}}=Q_{i}
$$

The dissipation function $D$ and the mixed collective potential $\mathscr{P}$ are defined as before [1] by

$$
D=\int_{\Omega}\left(\mathscr{D}_{\mathrm{v}}+\mathscr{D}_{\mathrm{tm}}\right) d \Omega, \quad \mathscr{P}=\int_{\Omega}(\mathscr{Y}+\rho \mathscr{G}) d \Omega,
$$

while the generalized boundary driving force $Q_{i}$ is given by

$$
Q_{i}=\int_{A}\left(\frac{\sigma_{j l}}{\rho} n_{l} \frac{\partial M_{j}}{\partial q_{i}}-\sum_{k} \varphi_{k} n_{j} \frac{\partial M_{j}^{k}}{\partial q_{i}}-\theta n_{j} \frac{\partial S_{j}}{\partial q_{i}}\right) d A
$$

The integral is extended to the boundary $A$ with the unit normal $n_{i}$. The terms containing the dissipative stress are simpler in this result because we have used the approximate value (4.19) for $\mathscr{D}_{\mathrm{v}}$ based on the viscosity coefficients of the mixture instead of the intermolecular values in [1].

If the entropy produced $s^{*}$ is sufficiently small it may be neglected in describing the state of the system and the Lagrangian equations (6.6) provide a complete set for the unknowns $q_{i}$. If this is not the case the equations contain additional auxiliary unknowns $q_{i}^{\prime}$. They may be determined by writing the additional equation

$$
T s^{*}=2 \mathscr{D}_{\mathrm{v}}+2 \mathscr{D}_{\mathrm{tm}}+\sum_{\rho} \mathscr{R}_{\rho} \dot{\xi}_{\rho}
$$

which is then assumed to be verified at a sufficient number of suitably chosen points to provide a complete set of equations for $q_{i}$ and $q_{i}^{\prime}$. 
Again proceeding as earlier [1], we may express the Lagrangian equations in terms of the kinetic energy of the fluid:

$$
\mathscr{T}=\frac{1}{2} \int_{\Omega} \rho v^{2} d \Omega
$$

Eqs. (6.6) become

$$
\frac{d}{d t}\left(\frac{\partial \mathscr{T}}{\partial \dot{q}_{i}}\right)-\frac{\partial \mathscr{T}}{\partial q_{i}}+\mathscr{M}_{i}+R_{i}+\frac{\partial D}{\partial \dot{q}_{i}}+\frac{\partial \mathscr{P}}{\partial q_{i}}=Q_{i}^{\prime}
$$

where

$$
\begin{aligned}
Q_{i}^{\prime}=Q_{i} & -\frac{1}{2} \int_{A} v^{2} n_{j} \frac{\partial M_{j}}{\partial q_{i}} d A, \\
\mathscr{M}_{i} & =\int_{\Omega} \mathscr{A}_{j} \frac{\partial M_{j}}{\partial q_{i}} d \Omega, \\
\mathscr{A}_{i} & =2 v_{j} \omega_{i j}, \\
\omega_{i j} & =\frac{1}{2}\left(\frac{\partial v_{i}}{\partial x_{j}}-\frac{\partial v_{j}}{\partial x_{i}}\right) .
\end{aligned}
$$

Due to the use of the barycentric velocity these expressions are simpler than those derived earlier for the nonreacting mixture [1].

In the particular case of small perturbations from equilibrium, the generalized affinity $R_{i}$ may be absorbed in the dissipation function $D$, while $\mathscr{M}_{i}$ is a higher-order quantity and $Q_{i}^{\prime}=Q_{i}$ to the first order. Hence the Lagrangian equations become

$$
\frac{d}{d t}\left(\frac{\partial \mathscr{T}}{\partial \dot{q}_{i}}\right)-\frac{\partial \mathscr{T}}{\partial q_{i}}+\frac{\partial D}{\partial q_{i}}+\frac{\partial \mathscr{P}}{\partial q_{i}}=Q_{i}
$$

These equations coincide with the general form of the Lagrangian equations of linear thermodynamics.

7. Evaluation of the thermodynamic functions. It is assumed that the equations of state of the mixture are known as

$$
p=p\left(v, m_{k}, T\right), \quad p_{k}=p_{k}\left(v, m_{l}, T\right)
$$

where $m_{k}$ are the masses of the various substances in the mixture of volume $v$ and pressure $p$. The injection pressure of each substances in the mixture is $p_{k}$, and $T$ is the temperature. These equations of state are obtained experimentally or derived theoretically from kinetic theories. Elimination of $v$ between them yields

$$
p_{k}=p_{k}\left(p, m_{k}, T\right) \text {. }
$$

The entropy differential of each pure substance per unit mass is

$$
d \bar{s}_{k}=\left(\frac{\partial \bar{s}_{k}}{\partial \bar{v}_{k}}\right)_{T} d \bar{v}_{k}+\left(\frac{\partial \bar{s}_{k}}{\partial T}\right)_{\bar{v}_{k}} d T
$$

where $\bar{v}_{k}$ is the specific volume. By definition of the specific heat $\bar{c}_{v}^{k}$ at constant volume, and 
from the classical Maxwell relations we may write

$$
\left(\frac{\partial \bar{s}_{k}}{\partial T}\right)_{\bar{v}_{k}}=\frac{\bar{c}_{v}^{k}}{T}, \quad\left(\frac{\partial \bar{s}_{k}}{\partial \bar{v}_{k}}\right)_{T}=\left(\frac{\partial p_{k}}{\partial T}\right)_{\bar{v}_{k}} .
$$

With these values,

$$
d \bar{s}_{k}=\left(\frac{\partial p_{k}}{\partial T}\right)_{\bar{v}_{k}} d \bar{v}_{k}+\frac{\bar{c}_{v}^{k}}{T} d T .
$$

The equation of state $p_{k}=p_{k}\left(\bar{v}_{k}, T\right)$ of the pure substance yields $\left(\partial p_{k} / \partial T\right)_{\bar{v}_{k}}$. With this value (7.5) of $d \bar{s}_{k}$ in Eqs. (2.4) we derive $\bar{s}_{k}$ and $\bar{\varepsilon}_{k}$ as functions of $p_{k}$ and $T$. Using expression (7.1) for $p_{k}$ we then obtain $\bar{s}_{k}\left(v, m_{l}, T\right)$ and $\bar{\varepsilon}_{k}\left(v, m_{l}, T\right)$ in terms of $v, m_{l}$ and $T$. The value of the heat of mixing $h_{p T}^{k}$ is obtained from the equation of state (7.1) for $p_{k}$ by using the formula (2.20).

We may now derive the values of the entropy $\mathscr{S}$ and energy $\mathscr{U}$ of a primary cell $C_{p}$ of volume $v$ containing a mixture. They are defined as collective concepts in the hypersystem, constituted by the cell $C_{p}$, supply cells $C_{s k}$, a chemical cell $C_{\mathrm{eq}}$, and a thermal well.

In the chemical cell $C_{\text {eq }}$, a chemical reaction of coordinate $\xi$ may occur at chemical equilibrium at the temperature $T_{\text {eq }}$. We start with a primary cell of zero volume, temperature $T$, and given concentrations. We then inject substances into it maintaining constant the concentrations, the pressure $p$ and the temperature $T$. This may be accomplished by thermobaric and thermal transfer. The chemical cell is made to produce masses $v_{k} \xi$ which are extracted gradually while maintaining constant the temperature $T_{\text {eq }}$. They are injected into the primary cell. At the same time masses $M^{k}$ are transferred from the supply cells into the primary cell in order to maintain constant the concentration. During this process the pressure $p$ of the primary cell is kept constant while sufficient heat of suitable sign is injected into $C_{p}$ and $C_{\mathrm{eq}}$ in order to maintain constant their respective temperatures. At the end of the process the state of the cell $C_{\mathrm{eq}}$ has remained unchanged and the state of the system $C_{\mathrm{eq}}+\sum_{k} C_{s k}+C_{p}$ is the same as if a reaction $\xi$ had occurred in $C_{p}$ and masses $M^{k}$ had been transferred to $C_{p}$ from $\sum_{k} C_{s k}$. The volume of the cell $C_{p}$ has increased from zero to $v$.

Actually the formulation is simplified by first transferring the masses $v_{k} \xi$ from the chemical cell to the supply cells $C_{s k}$ and from there to $C_{p}$. The total masses which have been transferred from $C_{s k}$ to $C_{p}$ are $m_{k}=v_{k} \xi+M^{k}$. Applying Eqs. (2.14) and (3.9), with a change of sign of $d \xi$, the differential increase $d \mathscr{S}$ of entropy of the system $C_{\text {eq }}+\sum_{k} C_{s k}+C_{p}$ during the process is

with

$$
d \mathscr{S}=\mathscr{S}_{\text {eq }} d \xi+\sum_{k} \mathscr{S}_{k} d m_{k}
$$

$$
\mathscr{S}_{\text {eq }}=\frac{\bar{h}_{p}^{\mathrm{eq}}}{T_{\text {eq }}}-\sum_{k} v_{k} \bar{s}_{k}^{\mathrm{eq}}, \quad \mathscr{S}_{k}=\bar{s}_{k}+\frac{h_{p T}^{k}}{T},
$$

where $\bar{s}_{k}^{\text {eq }}$ denotes the value of $\bar{s}_{k}$ for the chemical cell. Since all intensive variables are constant during the process this is also the case for $\mathscr{S}_{\text {eq }}$ and $\mathscr{S}_{k}$. Hence we obtain

$$
\mathscr{S}=\mathscr{S}_{\text {eq }} \xi+\sum_{k} \mathscr{S}_{k} m_{k}
$$

where $m_{k}$ are the masses in $C_{p}$. The quantity $\mathscr{S}$ defines the entropy of $C_{p}$.

Similarly the differential of the energy $\mathscr{U}$ is

$$
d \mathscr{U}=\mathscr{U}_{\mathrm{eq}} d \xi+\sum_{k} \mathscr{U}_{k} d m_{k}-p d v
$$


where

$$
\mathscr{U}_{\text {eq }}=\bar{h}_{p T}^{\text {eq }}-\sum_{k} v_{k} \bar{\varepsilon}_{k}^{\mathrm{eq}}, \quad \mathscr{U}_{k}=\bar{\varepsilon}_{k}+h_{p T}^{k},
$$

and $\bar{\varepsilon}_{k}^{\text {eq }}$ is the value of $\bar{\varepsilon}_{k}$ for the chemical cell. Since $p, \mathscr{U}_{\text {eq }}$ and $\mathscr{U}_{k}$ are also constant during the injection process we obtain for the energy of $C_{p}$

$$
\mathscr{U}=\mathscr{U}_{\text {eq }} \xi+\sum_{k} \mathscr{U}_{k} m_{k}-p v .
$$

The cell potential $\mathscr{V}$ is then derived from (2.1).

Using the values (2.1), (3.16), (7.8) and (7.11) we may verify the relation

$$
\mathscr{U}=-A \xi+\sum_{k} \phi_{k} M^{k}+\mathscr{S} T-p v
$$

in which we may use either values (3.16) or (3.23) for the affinity.

Similarly, using relations (7.6) and (7.9) we derive

$$
d \mathscr{U}=-A d \xi+\sum \phi_{k} d M^{k}+T d \mathscr{S}-p d v .
$$

For a cell of unit volume we put $v=1$. In this case $d v=0$ and Eq. (7.13) coincides with (3.32).

For simplicity we have assumed a single reaction; however, the results are readily generalized to multiple reactions, as in Eq. (3.32).

For a mixture of perfect gasses without chemical reactions, hence for $\xi=h_{p T}^{k}=0$, we note the following additive property.

$$
\mathscr{S}=\sum_{k} \bar{s}_{k} m_{k}, \quad \mathscr{U}+p v=\sum_{k} \bar{\varepsilon}_{k} m_{k} .
$$

The terms $\bar{s}_{k} m_{k}$ and $\bar{\varepsilon}_{k} m_{k}$ are respectively the entropy and enthalpy of the mass $m_{k}$ of the component gas, occupying alone the volume of the mixture at the same temperature.

8. Reformulation and generalization of the Gibbs-Duhem theorem. In the present context using the convective potentials $\phi_{k}$ and the collective definition of the entropy $\mathscr{S}$, the classical Gibbs-Duhem theorem requires a reformulation. We differentiate the values (7.8) and (7.11) of $\mathscr{U}$ and $\mathscr{S}$, and obtain

$$
\begin{aligned}
d \mathscr{S} & =\mathscr{S}_{\mathrm{eq}} d \xi+\sum_{k} \mathscr{S}_{k} d m_{k}+\sum_{k} m_{k} d \mathscr{S}_{k}, \\
d \mathscr{U} & =\mathscr{U}_{\mathrm{eq}} d \xi+\sum_{k} \mathscr{U}_{k} d m_{k}+\sum_{k} m_{k} d \mathscr{U}_{k}-p d v-v d p .
\end{aligned}
$$

Taking into account the values (7.6) and (7.9) of the differentials, this yields

$$
\sum_{k} m_{k} d \mathscr{S}_{k}=0, \quad \sum_{k} m_{k} d \mathscr{U}_{k}-v d p=0 .
$$

From these equations we derive

$$
\sum_{k} m_{k}\left(d \mathscr{U}_{k}-T d \mathscr{S}_{k}\right)=v d p .
$$

Evaluation of $d \mathscr{U}_{k}$ and $d \mathscr{S}_{k}$ from the expressions (7.7) and (7.10) yields

$$
d \mathscr{U}_{k}-T d \mathscr{S}_{k}=d \bar{\varepsilon}_{k}-T d \bar{s}_{k}+\frac{h_{p T}^{k}}{T} d T .
$$


We substitute the value of $h_{p T}^{k} / T$ obtained from (7.7). Hence we may write

$$
d \mathscr{U}_{k}-T d \mathscr{S}_{k}=d \phi_{k}+\mathscr{S}_{k} d T
$$

and Eq. (8.3) becomes

We have put

$$
\sum_{k} m_{k} d \phi_{k}+\mathscr{S}^{c} d T=v d p
$$

$$
\mathscr{S}^{c}=\sum_{k} m_{k} \mathscr{S}_{k} .
$$

According to (7.8), it is the value $\mathscr{S}$ of the entropy for $\xi=0$, i.e. in the absence of chemical reactions. We shall call $\mathscr{S}^{c}$ the convective entropy. It may be considered as a state variable. Eq. (8.6) constitutes the reformulation and a generalized form of the Gibbs-Duhem theorem. It is expressed in terms of the convective potentials $\phi_{k}$ and the convective entropy $\mathscr{S}^{c}$ instead of the chemical potential $\mu_{k}$ and the entropy used in the classical form. The present formulation avoids the basic difficulty of the classical treatment which involves undetermined constants in $\mu_{k}$ and the entropy. For $\xi=0$, we substitute $\mathscr{S}_{c}=\mathscr{S}$ and obtain the result already derived earlier [7] in the absence of chemical reactions.

For a cell of unit volume we substitute $v=1$ and $m_{k}$ become the masses of the constituents per unit volume. In a continuum, using (8.6), the total pressure gradient is obtained as

$$
\frac{\partial p}{\partial x_{i}}=\sum_{k} m_{k} \frac{\partial \phi_{k}}{\partial x_{i}}+\mathscr{S}^{c} \frac{\partial T}{\partial x_{i}}
$$

With the value (2.18) of $d \phi_{k}$ and the value (8.7) of $\mathscr{S}^{c}$ this becomes

$$
\frac{\partial p}{\partial x_{i}}=\sum_{k} \frac{m_{k}}{\rho_{k}} \frac{\partial p_{k}}{\partial x_{i}}+\sum_{k} \frac{h_{p T}^{k}}{T} \frac{\partial T}{\partial x_{i}} .
$$

For a perfect gas mixture we have $h_{p T}^{k}=0$ according to Eq. (2.22). Also $m_{k}$ in this case is the mass of a unit volume of pure substance at the pressure $p_{k}$, hence $m_{k}=\rho_{k}$. Hence for perfect gases eq. (8.10) becomes

$$
\frac{\partial p}{\partial x_{i}}=\sum_{k} \frac{\partial p_{k}}{\partial x_{i}}
$$

which is immediately evident from Dalton's law.

9. Translational invariance of the dissipation function and verification of the total momentum equation. Consider again the thermomolecular dissipation function (4.13). It must be invariant under an arbitrary translation of velocity $v_{i}$. When such a translation is superimposed the mass flux increases from $\dot{M}_{i}^{k}$ to $\dot{M}_{i}^{k}+m_{k} v_{i}$. The entropy flux as given by (4.3) is

$$
\dot{S}_{i}=\sum_{k} \bar{s}_{k} \dot{M}_{i}^{k}+\frac{\dot{H}_{i}}{T}
$$

According to the remark at the end of Sec. 4 , we have put $\dot{S}_{i}^{t}=\dot{H}_{i} / T$ where $\dot{H}_{i}$ is the heat flux which must be injected through a fixed area in addition to the masses $\dot{M}_{i}^{k}$ in order to 
maintain the actual temperature field. In the case of a pure translation this heat flux is

$$
\dot{H}_{i}=\sum_{k} h_{p T}^{k} m_{k} v_{i} \text {. }
$$

Hence, when adding such a translation, the entropy flux $\dot{S}_{i}$ becomes

$$
\dot{S}_{i}+\sum_{k} m_{k}\left(\bar{s}_{k}+\frac{h_{p T}^{k}}{T}\right) v_{i}=\dot{S}_{i}+\mathscr{S}^{c} v_{i}
$$

where

$$
\mathscr{S}^{c}=\sum_{k} m_{k}\left(\bar{s}_{k}+\frac{h_{p T}^{k}}{T}\right)=\sum_{k} m_{k} \mathscr{S}_{k}
$$

is the convective entropy as given by (8.7).

As a consequence, translational invariance is obtained by writing the thermomolecular dissipation function as

$$
\mathscr{D}_{t m}=\frac{1}{2} \sum_{l k i} C^{l k}\left(\frac{\dot{M}_{i}^{l}}{m_{l}}-\frac{\dot{M}_{i}^{k}}{m_{k}}\right)^{2}+\frac{1}{2} \sum_{k i} C^{k}\left(\frac{\dot{M}_{i}^{k}}{m_{k}}-\frac{\dot{S}_{i}}{\mathscr{S}^{c}}\right)^{2} .
$$

The mass of each component per unit volume is $m_{k}$. This result completes the expression for $\mathscr{D}_{t m}$ derived in earlier work [1] and takes into account the correct dependence ${ }^{(1)}$ on $\dot{S}_{i}$. In accordance with the value (4.13) the values of $C_{k}$ must be chosen so that the coefficient of $\dot{S}_{i}^{l}$ is equal to $T / l k$, where $k$ is the combined kinetic and radiation conductivity. The coefficients $C^{l k}$ and $C^{k}$ are functions of the local state variables of the mixture, $m_{k}$ and $T$.

The dissipation function (9.5) satisfies for each $i$ the important identity,

$$
\sum_{k} m_{k} \frac{\partial \mathscr{D}_{t m}}{\partial \dot{M}_{i}^{k}}+\mathscr{S}^{c} \frac{\partial \mathscr{D}_{t m}}{\partial \dot{S}_{i}}=0
$$

Using this relation we may verify the total momentum equation as follows. Let us go back to the field equations (5.10). We multiply each equation of the first set by $m_{k}$ and the equation of the second set by $\mathscr{S}^{c}$, and add the results. Taking into account the identity (9.6) we obtain

$$
\rho a_{i}-\frac{\partial \sigma_{i j}}{\partial x_{j}}+\sum_{k} m_{k} \frac{\partial \phi_{k}}{\partial x_{i}}+\mathscr{S}^{c} \frac{\partial T}{\partial x_{i}}+\rho \frac{\partial \mathscr{G}}{\partial x_{i}}=0
$$

In deriving this equation we have introduced the value $\varphi_{k}=\phi_{k}+\mathscr{G}$ and put $\theta=T-T_{0}$. Using relation (8.9), derived from the Gibbs-Duhem theorem, Eq. (9.7) becomes

$$
\rho a_{i}-\frac{\partial \sigma_{i j}}{\partial x_{j}}+\frac{\partial p}{\partial x_{i}}+\rho \frac{\partial \mathscr{G}}{\partial x_{i}}=0
$$

which is obviously the total momentum equation.

10. Gas mixture with radiation pressure. The theory is immediately applicable to a gas mixture at high temperature with radiation pressure. In this case it is convenient to write the total pressure $p$ as the sum of the molecular pressure $p^{\mathrm{mol}}$ and the radiation pressure $p^{\mathrm{rad}}$ :

$$
p\left(m_{k}, T\right)=p^{\mathrm{mol}}\left(m_{k}, T\right)+p^{\mathrm{rad}}\left(m_{k}, T\right) .
$$

(1) Note that the value $\mathscr{D}_{t m}$ in [1] is valid only for $h_{p T}^{k}$ negligible. 
They are expressed as functions of $T$ and the masses $m_{k}$ of each substance per unit volume. Similarly we write the injection pressure as

$$
p_{k}\left(\rho_{k}, T\right)=p_{k}^{\mathrm{mol}}\left(\rho_{k}, T\right)+p_{k}^{\mathrm{rad}}\left(\rho_{k}, T\right) .
$$

In vacuum the radiation pressure is [15]

$$
p^{\mathrm{rad}}=p_{k}^{\mathrm{rad}}=\frac{1}{3} \sigma T^{4}
$$

where $\sigma$ is Stefan's constant. However, as pointed out by Brillouin [16], for a dense mixture the radiation pressures depend on $m_{k}$ and $\rho_{k}$. This is due to the fact that the group velocity of the radiation in this case is not equal to the velocity of light in vacuum.

Using the equations of state (10.1) and (10.3) we may derive all the thermodynamic functions by the procedure outlined in Sec. 7. Note that in Eq. (7.5) the specific heat $\bar{c}_{v}^{k}$ may be written as the sum of a molecular term and a radiation term

$$
\bar{c}_{v}^{k}=\bar{c}_{v}^{k(\mathrm{~mol})}+\bar{c}_{v}^{k(\mathrm{rad})} .
$$

Let us consider the particular case where the molecular pressures are the same as for a perfect gas while the radiation pressure is the vacuum value (10.3). In this case, for a volume $v$ of mixture

$$
p^{\mathrm{mol}} v=\sum_{k} n_{k} R T, \quad p_{k}^{\mathrm{mol}} v=n_{k} R T,
$$

where $n_{k}$ is the number of moles of each gas in the volume $v$ and $R$ is the gas constant. Also,

$$
p=p^{\mathrm{mol}}+\frac{1}{3} \sigma T^{4}, \quad p_{k}=p_{k}^{\mathrm{mol}}+\frac{1}{3} \sigma T^{4} .
$$

From (10.5) and (10.6) we obtain, for the injection pressure, the value

$$
p_{k}=\gamma_{k} p+\frac{1}{3} \sigma T^{4}\left(1-\gamma_{k}\right)
$$

where $\gamma_{k}=n_{k} / \sum_{k} n_{k}$ is the molar fraction of each gas in the mixture. With this value of $p_{k}$ Eq. (2.20) yields

$$
h_{p T}^{k}=\frac{4}{3 \rho_{k}} \sigma T^{4}\left(\gamma_{k}-1\right) .
$$

Hence the mixture in this case does not behave as a perfect gas since $h_{p T}^{k} \neq 0$.

Taking into account the radiation heat $\sigma T^{4}$ per unit volume, the specific heat (10.4) becomes

$$
\bar{c}_{v}^{k}=\bar{c}_{v}^{k(\mathrm{~mol})}+\frac{4 \sigma T^{3}}{\rho_{k}}
$$

where $\bar{c}_{v}^{k(\mathrm{~mol})}$ is the molecular specific heat of the perfect gas.

With these results the theory is immediately applicable to very general problems of stellar dynamics. It yields either the field equations for $M_{i}^{k}$ and $S_{i}$ or the Lagrangian equations of Sec. 6 where the problem is formulated by means of generalized coordinates. The latter method should provide a particularly powerful approach to problems of star oscillations with or without spherical symmetry. Actually, of course, the effect of ionization may also be taken into account by including the ionization energy in the chemical parameters. 


\section{REFERENCES}

[1] M. A. Biot, New variational-Lagrangian thermodynamics of viscous fluid mixtures with thermomolecular diffusion, Proc. R. Soc. London A.365, 467-494 (1979)

[2] G. W. Gibbs, Thermodynamics, vol. I, Longmans, London (1906)

[3] G. N. Hatsopoulos and J. H. Keenan, Principles of general thermodynamics, John Wiley \& Sons Inc. New York (1965)

[4] I. Prigogine and R. Defay, Chemical thermodynamics, Longman, London (1954)

[5] M. A. Biot, Variational-Lagrangian irreversible thermodynamics of initially stressed solids with thermomolecular diffusion and chemical reactions, J. Mech. Phys. Solids 25, 289-307 (Errata 1978, 26, 59) (1977)

[6] M. A. Biot, New chemical thermodynamics of open systems: Thermobaric potential-a new concept, Bull. Acad. R. Belg. (Cl. Sc.) 62, 239-258 (Erratum, 62, 678) (1976)

[7] M. A. Biot, New fundamental concepts in thermodynamics with chemical applications, Chem. Phys. 22, 183198 (1977)

[8] Th. De Donder, L'Affinité, Gauthiers-Villars, Paris (1934)

[9] M. A. Biot, Variational irreversible thermodynamics of physical-chemical solids with finite deformation, Int. J. Solids Structures 14, 881-903 (1978)

[10] M. A. Biot, Variational-Lagrangian thermodynamics of evolution of collective chemical systems, Chem. Phys. 29,97-115(1978)

[11] R. Fowler and E. A. Guggenheim, Statistical thermodynamics, Cambridge University Press (1952)

[12] L. Onsager, Reciprocal relations in irreversible processes I, Phys. Rev. 37, 405-426(1931)

[13] L. Onsager, Reciprocal relations in irreversible processes II, Phys. Rev. 38, 2265-2279 (1931)

[14] I. Müller, On the frame dependence of stress and heat flux, Arch. Rat. Mech. Anal. 45, 241-250(1972)

[15] Y. Rocard, Thermodynamique, Masson, Paris (1952)

[16] L. Brillouin, Les statistiques quantiques et leurs applications, Presses Universitaires de France, Paris (1930) 